\title{
Targeting EGFR signalling in chronic lung disease: therapeutic challenges and opportunities
}

\author{
Sabari Vallath, Robert E. Hynds, Laura Succony, Sam M. Janes and \\ Adam Giangreco
}

\section{Affiliations:}

Lungs for Living Research Centre, Division of Medicine, University College London, UK.

\section{Correspondence:}

Adam Giangreco, Lungs for Living Research Centre, Division of Medicine, University College London, 5 University Street, London, WC1E 6JF, UK.

E-mail: a.giangreco@ucl.ac.uk

\begin{abstract}
Chronic respiratory diseases, including pulmonary fibrosis, chronic obstructive pulmonary disease (COPD) and lung cancer, are the second leading cause of death among Europeans. Despite this, there have been only a few therapeutic advances in these conditions over the past 20 years. In this review we provide evidence that targeting the epidermal growth factor receptor (EGFR) signalling pathway may represent a novel therapeutic panacea for treating chronic lung disease. Using evidence from human patient samples, transgenic animal models, and cell and molecular biology studies we highlight the roles of this signalling pathway in lung development, homeostasis, repair, and disease ontogeny. We identify mechanisms underlying lung EGFR pathway regulation and suggest how targeting these mechanisms using new and existing therapies has the potential to improve future lung cancer, COPD and pulmonary fibrosis patient outcomes.
\end{abstract}

@ERSpublications

Deregulated EGFR pathway signalling is a common event and an important therapeutic target for many chronic lung diseases http://ow.ly/rH74p

\footnotetext{
For editorial comments see page 297.

Received: Aug 212013 | Accepted after revision: Dec 012013 | First published online: Jan 162014

Conflict of interest: None declared.

Copyright @ERS 2014
} 


\section{Introduction}

Chronic respiratory diseases, including pulmonary fibrosis, chronic obstructive pulmonary disease (COPD) and lung cancer, cost European healthcare systems in excess of $€ 100$ billion per annum and together represent the second most common cause of death among Europeans [1]. Despite this significant disease burden, there have been few therapeutic breakthroughs and mortality rates for these conditions have remained largely unchanged for many years [2]. In contrast, research conducted over the past decade has dramatically improved our understanding of the genomic and signalling pathway changes associated with chronic respiratory disease [3-7]. These advances have allowed clinicians to investigate whether stratifying and treating patients based on the presence of specific mutations might improve disease outcomes. Unfortunately, this research has uncovered extensive heterogeneity present within and among most respiratory diseases. These data suggest that an additional understanding of which mutations are responsible for respiratory disease initiation will be needed to provide a more tractable approach for improving patient survival [8].

The epidermal growth factor receptor (EGFR) signalling pathway is a strong contender for both initiating and determining clinical outcomes in many respiratory diseases. Deregulation of the EGFR pathway causing aberrant EGFR signalling is associated with the early stage pathogenesis of lung fibrosis, cancer and numerous airway hypersecretory diseases, including COPD, asthma and cystic fibrosis [9-11]. In this review, we provide evidence that targeting the EGFR signalling pathway may represent a novel therapeutic approach for treating these multiple lung diseases. We describe the mechanisms underlying EGFR pathway regulation in normal and diseased airways and suggest that targeting these using established and indevelopment therapies has the potential to improve outcomes in chronic respiratory disease.

\section{The EGFR signalling pathway}

The EGFR signalling pathway is complex and comprises four cell surface receptors, at least 13 extracellular ligands, and four key downstream effector pathways that regulate a wide variety of cellular functions (fig. 1) [12]. Epidermal growth factor (EGF) receptors include HER1 (EGFR/ErbB1), HER2 (ErbB2), HER3 (ErbB3) and HER4 (ErbB4), all of which contain a cysteine-rich extracellular ligand binding domain, a single-pass $\alpha$-helix transmembrane domain, a C-terminal signalling domain and, with the exception of HER3, a cytoplasmic tyrosine kinase domain [13]. Ligand-receptor binding causes dimerisation either with another receptor of the same type (homodimerisation) or with a different member of the EGFR family (heterodimerisation) [14, 15]. EGFR dimerisation then activates one or more downstream effectors including the MEK/ERK (MAPK kinase/extracellular signal-regulated kinase), PI3K/AKT (phosphatidylinositol-3-kinase/protein kinase B), STAT (signal transducer and activator of transcription), and mTOR (mammalian target of rapamycin) pathways through receptor autophosphorylation and cytoplasmic protein binding [16]. These in turn act as critical mediators of airway and alveolar homeostasis, with aberrant activation within one or more pathway components capable of driving a variety of respiratory pathologies. Common pathological outcomes associated with EGFR pathway misactivation include excessive airway proliferation, mucus overproduction and hypersecretion, and progressive distal lung fibrosis (fig. 1).

Given the complexity of the EGFR signalling pathway, it is perhaps unsurprising that the diversity of outcomes following receptor activation is mediated through a variety of methods and control points. These include ligand choice and binding affinity, receptor homo- and/or heterodimerisation, extracellular ligand release and physical segregation of ligands and receptors, accessibility and abundance of intracellular and cell surface antagonists, speed and capacity for receptor recycling, and choice of downstream signalling pathway and subsequent gene regulation (fig. 2).

Functional selectivity, or divergent activation of downstream pathways by different ligands binding to the same receptor, may be mediated by subtle differences in the conformation of ligand-receptor complexes [17]. All EGF-family ligands are synthesised in a membrane-docked form that must be further processed in order to release biologically active forms [18]. These cleaved proteins contribute to the extracellular signalling environment, either directly through the activation of cell surface receptors or through binding to proteoglycans in the extracellular matrix to form a reservoir of growth factors that can later be freed by extracellular proteases. Membrane-anchored ADAMs (disintegrin metalloproteases) are key regulators of EGFR ligand release. For example, ADAM10 is known to cleave EGF and betacellulin, while ADAM17 is considered the primary cleavage enzyme for many other EGF family ligands [19, 20]. Ligand cleavage can also occur via matrix metalloproteinases. For example, HER1 is transactivated upon stimulation of Gprotein-coupled receptors because HB-EGF is released from its pro form [21] via the formation of a cell surface signalling complex including MMP7 [22]. 


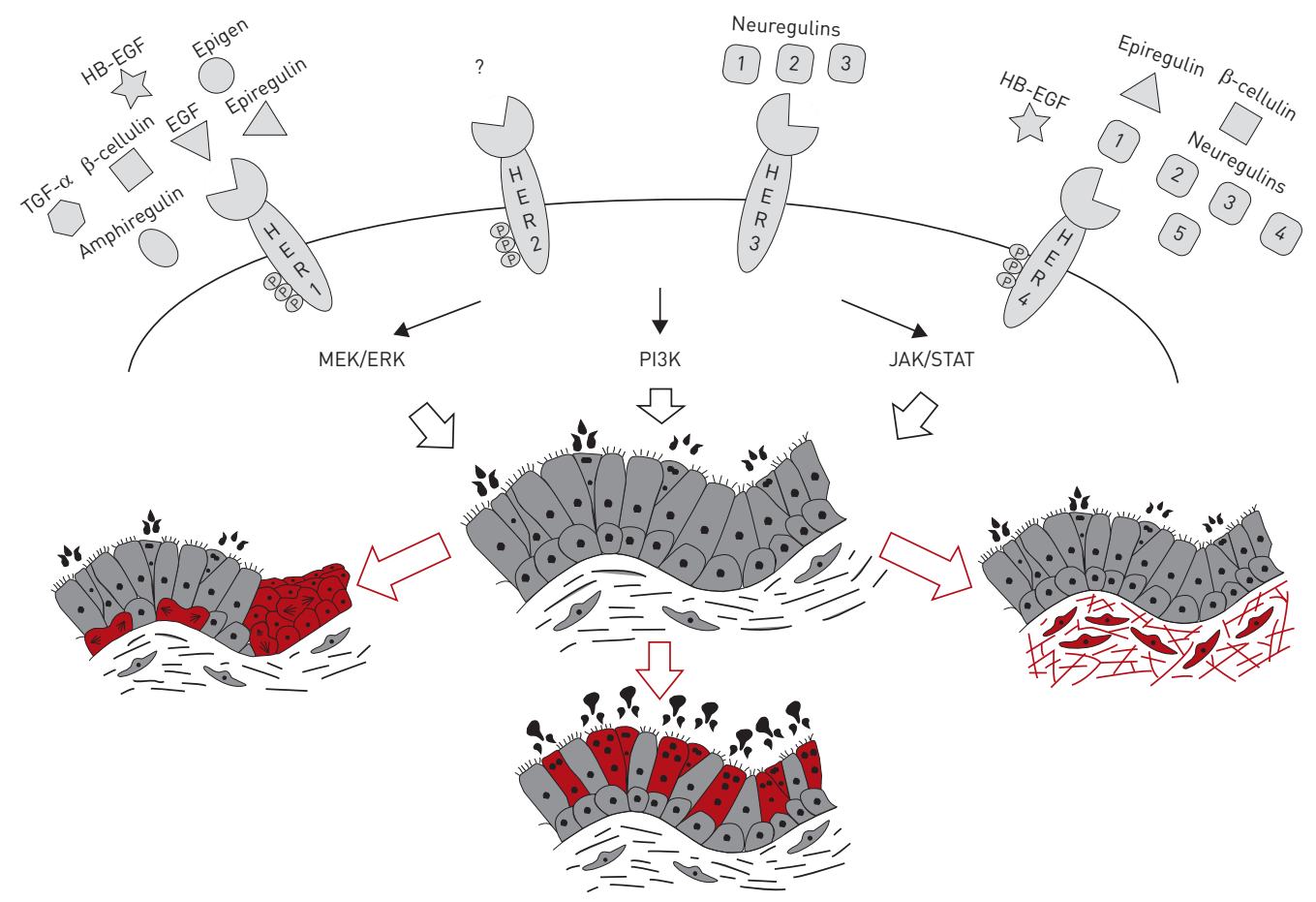

FIGURE 1 A simplified view of the epidermal growth factor receptor (EGFR) signalling pathway in lung homeostasis and disease. The HER family comprises four members, each of which is activated by one or more of the 13 identified members of the epidermal growth factor (EGF) family of ligands (shown above each receptor). Receptor-ligand interactions lead to autophosphorylation of the receptor intracellular domain and the recruitment of downstream signalling components. Effectors of EGFR signalling include the MEK/ERK (MAPK kinase/extracellular signal-regulated kinase), PI3K (phosphatidylinositol-3-kinase) and JAK/STAT (Janus kinase/signal transducer and activator of transcription) pathways, whose combined activity regulates airway development and homeostasis. Healthy airways are lined by a tightly regulated epithelial barrier comprised of basal, mucosecretory and ciliated cell populations. In chronic respiratory disease, aberrant EGFR signalling can cause airway hyperproliferation (in red, left), increase mucus cell differentiation and mucus production (red, centre) and promote subepithelial fibrosis and excessive collagen deposition (red, right). TGF: transforming growth factor.

Receptor and ligand segregation provides an additional mechanism for regulating EGFR signalling [23, 24]. In airways, ligands produced apically are physically prevented from interacting with their receptors present basolaterally on different cell types. When the epithelium is compromised, cell-cell contact is disturbed and ligands interact with normally inaccessible receptors on the basolateral membrane of airway basal cells [23, 24]. The subsequent activation of HER family receptors engages signalling pathways that ensure proliferation occurs and barrier integrity is rapidly restored.

As well as these physical means of EGFR pathway control, several endogenous EGFR pathway antagonists have been identified. LRIG1 is a plasma membrane protein expressed to varying levels within airway, skin and intestinal epithelial cells that acts as an inhibitor of EGFR pathway-dependent proliferation [25-28]. Data suggests that LRIG1 acts in airways by recruiting a HER1:E-Cadherin tripartite complex upon cellular confluency [28]. Whereas LRIG1 is membrane-bound, the HER1 antagonist MIG6 is a separate, cytoplasmic protein that inhibits the tyrosine kinase domain of HER family receptors to prevent their dimerisation and subsequent kinase activity [29].

Upon ligand binding and pathway activation, EGF receptors are rapidly internalised to prevent continuous receptor activation. Given the aberrant effects of over-activation of the EGFR pathway, tight regulation of receptor phosphorylation occurs under normal conditions [30]. HER1 autophosphorylation can be reversed directly by Tyr-specific phosphatases that dephosphorylate the kinase domain, preventing further signalling. Additionally, once receptors are activated, cells internalise them to dissociate the ligand source and receptor, preventing continued signalling. The recruitment of adaptor proteins, such as growth factor receptor-bound protein 2 (GRB2), facilitate clathrin-mediated endocytosis of the receptor and in turn recruit ubiquitin ligases [31]. Ubiquitination targets the receptor for degradation via the lysosomal pathway but deubiquitylating enzymes can intervene at this stage to prevent degradation and recycle the receptor to the cell surface [32]. The fate of internalised EGF family receptors is also ligand-dependent; some ligands, 


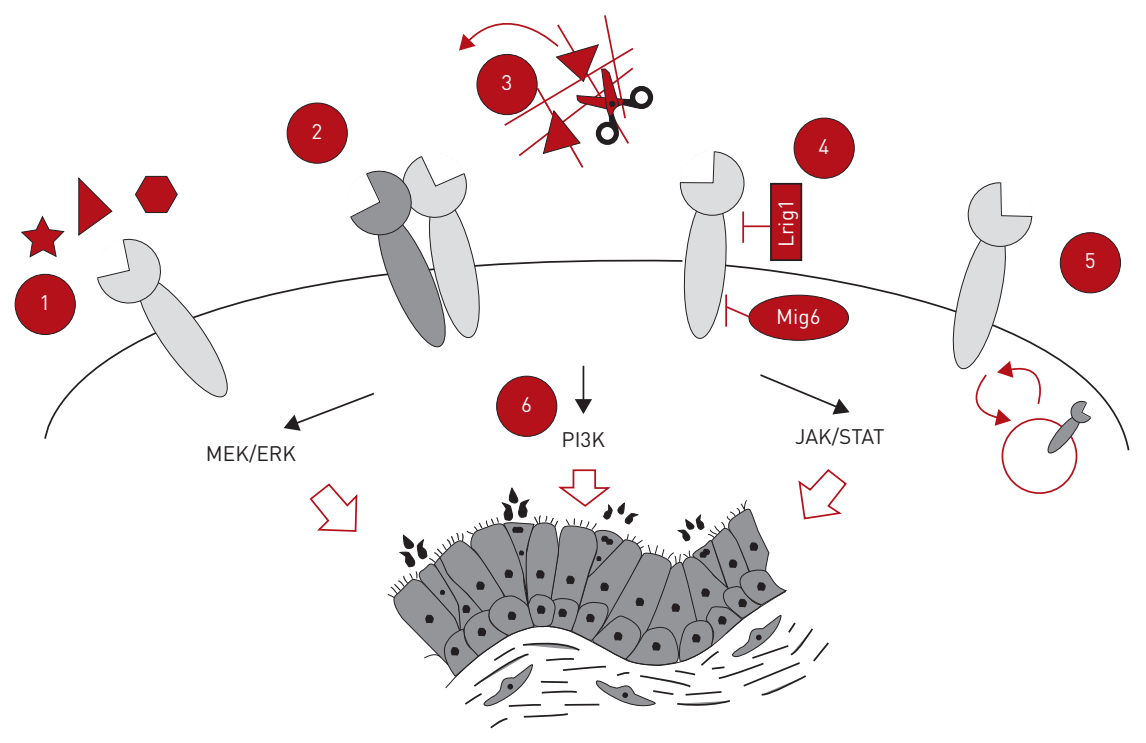

FIGURE 2 Mechanisms of endogenous epidermal growth factor receptor (EGFR) signalling pathway regulation. During homeostasis several endogenous mechanisms exist to modulate EGFR pathway activity. These include 1) ligand-receptor choice and binding affinity, 2) EGFR homo- and heterodimerisation, 3) extracellular ligand release and receptor-ligand segregation, 4) cytoplasmic and cell surface antagonist abundance, 5) rate of intracellular receptor recycling, and 6) downstream pathway choice causing differential gene expression. MEK/ERK: MAPK kinase/extracellular signal-regulated kinase; PI3K: phosphatidylinositol-3-kinase; JAK/STAT: Janus kinase/signal transducer and activator of transcription.

such as EGF, remain tightly bound and receptors continue through the endosomal pathway and are ultimately lysosomally degraded while others, such as transforming growth factor (TGF)- $\alpha$, dissociate more readily, favouring receptor recycling $[33,34]$.

\section{EGFR signalling in lung development and homeostasis}

Although normal airway HER1 expression is low and only transiently increased during repair [35], there nonetheless exists a significant body of evidence indicating a fundamental role for EGFR signalling in lung development and homeostasis. Specifically, mice lacking Erbb1 (human HER1) exhibit impaired lung development, lung branching deficiencies from as early as E12.5, and invariably die in utero [36]. Conversely, constitutive Erbb1 pathway activation disrupts normal lung morphogenesis and promotes phenotypes reminiscent of chronic lung disease [35, 37]. Mice that overexpress the Erbb1 (human HER1) ligand TGF- $\alpha$ in their airways develop fibrosis, pleural thickening, abnormal vascular development and pulmonary hypertension, a phenotype that can be ablated by cross breeding with Erbb1 mutant mice. These studies have also shown that mice deficient in TGF- $\alpha$ are resistant to bleomycin-induced lung remodelling [38]. Further, in the alveolar epithelium, EGF signalling via Erbb1 has emerged a key regulator of type II cell maturation and mediator of acute lung injury responses [39, 40]. HER2 and HER3 are additionally known to be expressed during human lung development [41] and HER4 signalling regulates epithelial proliferation [42] and surfactant production $[43,44]$ in fetal lungs. Together, these results point to EGFR signalling as a critical developmental pathway in lung epithelial cells.

Murine data regarding Erbb1 in lung development is supported by in vitro work on human airway cells that suggests a key role for HER1 signalling in normal lung homeostasis. In human airway air-liquid interface models, increasing the concentration of neurgulin-1 in cultures increases epithelial height [45] while increasing trefoil factor family 3 (TFF3) concentration leads to HER1 pathway-dependent increase in the proportion of epithelial cells adopting a ciliated phenotype [46]. The addition of EGF or other EGF family ligands to these cultures also promotes squamous-like epithelial differentiation with decreased ciliated cell differentiation and acquisition of an epithelial-to-mesenchymal transition phenotype [24]. These results suggest that EGFR signalling is important for maintenance of normal human airway homeostasis.

\section{EGFR signalling in lung fibrosis}

The EGFR pathway has been implicated in lung fibrosis through studies in which transgenic mice that constitutively express TGF- $\alpha$ in epithelial cells develop progressive lung fibrosis [35, 37, 47]. This occurs via an EGFR pathway dependent paracrine loop between epithelial and fibroblast cells, resulting in excessive collagen production and deposition. In this model, even transient increases in TGF- $\alpha$ expression disrupt 
lung morphogenesis, arrest normal alveolar septation, and cause chronic lung disease [35]. Conversely, mice deficient in TGF- $\alpha$ that lack normal EGFR signalling (wa-2 mutant mice) or that are treated with EGFR pathway antagonists exhibit resistance to bleomycin-induced lung fibrosis [38]. It has also been shown that the HER1 ligand amphiregulin and an intact EGFR pathway are required for TGF- $\beta 1$ dependent pulmonary fibrosis [48]. The administration of bleomycin to mice is an established lung fibrosis model. Administering the tyrosine kinase inhibitor gefitinib with bleomycin attenuated these changes. There was evidence that the bleomycin-induced phosphorylation of EGFR was inhibited by gefitinib [49]. These data suggest a novel, under-appreciated clinical relevance of inhibiting EGFR signalling in regulating human fibrotic lung disease.

\section{EGFR signalling in airway hypersecretory disease}

Excessive mucus production and secretion is a pathological feature of a number of chronic respiratory diseases, including asthma, COPD, and bronchiectasis. It is increasingly accepted that the EGFR signalling pathway is a central regulator of both airway mucus production and secretion. Specifically, a significant positive correlation exists between high airway EGFR immunoreactivity and excessive goblet cell differentiation in asthmatic patient airways [50], and bronchial biopsies obtained from smokers with excessive mucus production exhibit increased HER1 and HER3 expression [51]. Cystic fibrosis patients also exhibit evidence of increased mucin expression, TGF- $\alpha$ abundance and EGFR signalling in their airways [52]. Pseudomonas aeruginosa, a colonising bacterium, is associated with worsening outcomes in cystic fibrosis patients and can trigger EGFR activation and subsequent MUC5AC expression in vitro. These effects can be blocked by HER1 antagonism [53], suggesting that an EGFR-targeted therapy may be useful in future therapies seeking to reduce mucin load in cystic fibrosis.

Airway inflammation, with release of the pro-inflammatory cytokine tumour necrosis factor (TNF)- $\alpha$ has been shown to induce excessive HER1 signalling in airways [54], upregulate amphiregulin, and promote TGF- $\alpha$ secretion from bronchial epithelial cells $[55,56]$. These changes exert an autocrine effect on HER1 activity, leading to increased goblet cell differentiation and mucus secretion. Experimentally, this has been shown by the ability of intratracheal TNF- $\alpha$ instillation to promote HER1 expression in rat airways [57]. Instillation of EGFR pathway ligands also causes expansion of goblet cell populations and increased mucin production [58]. The pro-inflammatory cytokines that induce EGFR expression in diseased airways are most likely derived from immune cells: CD4+ T-cells produce a Th2 pattern of cytokines, including interleukin (IL)-4, IL-5 and IL-13 [59], that in turn stimulate airway epithelial cell proliferation through autocrine production of TGF- $\alpha$ [60]. Separately, activated eosinophils grown in the presence of inflammatory cytokines IL-3 and IL-5 can induce upregulation of TGF- $\alpha$ in bronchial epithelial cells, leading to HER1 activation and increased mucin production [61]. Pro-inflammatory cytokine release also occurs upon epithelial exposure to fine particulate matter, resulting in increased amphiregulin, TGF- $\alpha$ and EGF ligand expression as well as mucus production [62]. Altogether, these data suggest that antagonists of EGFR ligands, receptors, and/or downstream mediators may ameliorate excessive goblet cell differentiation and mucus production in a wide variety of hypersecretory diseases.

\section{EGFR signalling in lung cancer}

The EGFR signalling pathway plays an important role in multiple lung cancers. Aberrant EGFR pathway activation is found in essentially all head and neck cancer patients and conserved HER1 gene mutations have been identified in subsets of both squamous and adenocarcinoma patients [63, 64]. Of these mutations, approximately $90 \%$ of mutations are found in exons 18-21 of the HER1 kinase domain, with an additional 5\% represented by an in-frame deletion within exons 2-7 [9].

Given this clear importance of EGFR signalling in lung cancer, many groups have produced mouse models that mimic abnormal human lung HER1 expression. Erbb1 (HER1) mouse models that exhibit humanlike adenocarcinoma development include doxycycline-inducible hEGFR ${ }^{\mathrm{L} 858 \mathrm{R}}$ (CCSP-rtTA;Tet- ( $_{7}$ hEGFR $^{\text {L858R }}$ ), hEGFR ${ }^{\text {DEL }}$ (CCSP-rtTA;Tet-O -hEGFR $^{\text {DEL }}$ ), and hEGFR ${ }^{\text {VIII }}$ (CCSP-rtTA;TetO 7 -hEGFR ${ }^{\text {VIII }}$ ) lines [65]. In each case, mutant mice treated with doxycycline develop multifocal tumours in the lung parenchyma. Missense mutation (L858R) and deletion (DEL) lines treated with either small molecule HER1/2 tyrosine kinase inhibitors (gefitinib, erlotinib or HKI-272) or monoclonal antibody therapies (cetuximab) also exhibit complete regression of doxycycline-inducible tumours [66]. Separate animal models in which either the membrane associated HER1 suppressor LRIG1 or the intracellular regulator Mig6 are deleted also exhibit increased lung cancer susceptibility [28, 67-69]. These data are consistent with those from human clinical specimens in which both LRIG1 and MIG6 are downregulated in squamous and adenocarcinomas in conjunction with EGFR pathway activation [28]. Intriguingly, LRIG1 and MIG6 deletion models each also exhibit features of early preinvasive lung cancer, suggesting that EGFR family antagonism may be clinically relevant in the treatment of early lung disease, even in the absence of identified HER1/2 mutations $[28,70]$. 


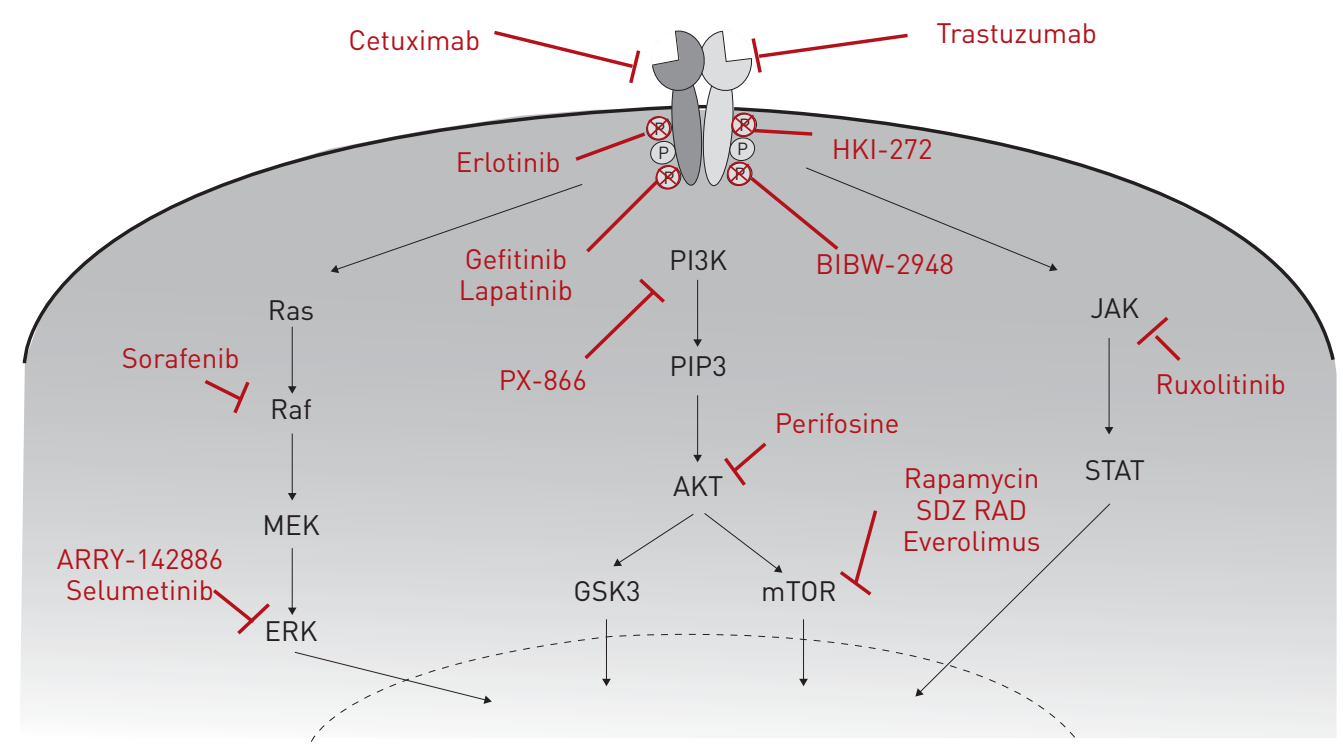

FIGURE 3 Clinically approved and in-development epidermal growth factor receptor (EGFR) signalling pathway inhibitors for chronic respiratory disease. Three strategies are used to antagonise aberrant EGFR signalling. First, monoclonal antibodies bind the extracellular domain of epidermal growth factor (EGF) receptors and prevent endogenous ligand engagement, thereby attenuating downstream pathway activation (cetuximab and trastuzumab). Secondly, small molecule tyrosine kinase inhibitors target the intracellular portion of EGF receptors; in the absence of kinase activity EGFR autophosphorylation and downstream signalling is inhibited (erlotinib, gefitinib, lapatinib, HKI-272 and BIBW-2948). Finally, small molecule inhibitors which target specific downstream EGFR signalling pathway components have also been developed and may exhibit clinical efficacy in chronic respiratory disease (ARRY-142886, PX866, rapamycin, SDZ RAD, everolimus, ruxolitinib, perifosine, selumetinib and sorafenib). PI3K: phosphatidylinositol-3kinase; PIP3: phosphatidylinositol (3,4,5)-triphosphate; Akt: protein kinase B; GSK3: glycogen synthase kinase 3; mTOR: mammalian target of rapamycin; MEK: MAPK kinase; ERK: extracellular signal-regulated kinase; JAK: Janus kinase; STAT: signal transducer and activator of transcription.

\section{Therapeutically targeting the EGFR pathway}

To date the majority of clinical studies involving EGFR pathway inhibition have focused on treatments for lung cancer where mutations in either HER1 or HER2 have been identified. There are at present two main therapeutic approaches used to inhibit mutated EGFR pathway signalling: small molecule tyrosine kinase inhibitors (TKIs) targeting intracellular EGFR signalling molecules and monoclonal antibody therapies directed at blocking receptor-ligand binding (fig. 3).

Small molecule TKIs have been licensed for HER1 mutation positive lung cancer for a number of years, with their unequivocal benefit shown in IPASS (the Iressa Pan-Asia Study) [71]. This phase III multicentre, panAsian, open label study randomised hitherto untreated patients with advanced nonsmall cell lung cancer to a first line TKI (gefitinib) versus conventional therapy consisting of carboplatin and paclitaxel. Patients positive for HER1 mutations showed a significant objective response rate with a TKI treatment response of $71.2 \%$ versus $1.1 \%$ with conventional chemotherapy [72]. Monoclonal antibody therapies, including cetuximab and trastuzumab, used to target HER1 and HER2 mutations in other diseases also showed potential utility in lung cancers. Sadly, in a phase II clinical trial of patients with identified HER1 mutations, these compounds have not proved clinically beneficial [73]. Additionally, the use of both TKIs and monoclonal antibodies are also often limited due to the acquisition and development of tumour therapy resistance [74]. Resistance to chemotherapeutic drugs is a key issue in lung cancer patient management and recent studies have linked it, at least in part, to the existence of cancer stem-like cells. These subpopulations of cells within the tumour possess stem cell-like features, are positive for CD133 and ALDH (aldehyde dehydrogenase family) and have been reported to show greater tumorigenic potential and higher resistance to chemotherapeutic/EGFR TKI drugs $[75,76]$. Studies have also shown that Met amplification together with sustained PI3K/AKT signalling pathway may confer TKI drug resistance in HER1/2 mutant tumours [77]. For this reason, alternative strategies, including the development of second generation TKIs and dual targeting of EGF receptors in conjunction with other EGFR pathway targets, are currently being investigated at a preclinical and clinical level [78]. These studies involve targeting novel downstream PI3K, AKT, JAK/ STAT and mTOR mediators in conjunction with EGF receptor inhibition (some of which are shown in fig. 3). Of note, mTOR inhibitors are currently approved for use in renal cell carcinoma [79], although their efficacy in lung cancer has not been established. 
In addition to lung cancer, recent studies targeting interactions between TGF- $\alpha$ and HER 1 in pulmonary fibrosis have shown significant preclinical success. In transgenic mice that overexpress TGF- $\alpha$ and develop spontaneous pulmonary fibrosis the use of the MEK inhibitor ARRY-142886 or the PI3K inhibitor PX-866 reduces pulmonary fibrosis progression and preserves physiological parameters [80, 81]. Administration of the mTOR inhibitor rapamycin in this same model also inhibits progression of established pulmonary fibrosis by limiting EGFR signalling pathway activation [82]. In a separate rat model of bleomycin-induced fibrosis the rapamycin analogue SDZ RAD reduced lung collagen accumulation and lung weights, both of which are classic features of lung fibrosis [83]. Clinically, the rapamycin analogue everolimus was assessed in a double blind placebo controlled trial of 88 patients with biopsy proven idiopathic pulmonary fibrosis. Disappointingly, almost half of the patients in the treatment arm of this study did not tolerate the trial dosing regimen and exhibited a decline in lung function parameters for unknown reasons [84]. Further clinical studies are therefore needed to assess the potential utility of targeting these and other AKT and JAK/ STAT inhibitors currently in development for other fibroproliferative disorders, such as ruxolitinib and perifosine (fig. 3) [85, 86].

Given the significant evidence that the EGFR pathway regulates airway mucus cell hyperplasia and mucus production, a number of preclinical studies have examined whether targeting this pathway might be useful in treating asthma, COPD, cystic fibrosis and other mucus hypersecretory disorders. In an animal model of mucus hypersecretion, blocking TGF- $\alpha$ using small molecule inhibitors prevented IL-13 dependent mucus production [87]. Separately, small molecule and monoclonal antibody EGFR inhibitors can prevent mucin upregulation in a number of preclinical models $[53,62,88]$. A clinical trial in which the EGFR TKI BIBW2948 was administered to COPD patients with mucus hypersecretion has also been established. This multicentre, double- blind, placebo-controlled trial, analysed safety and mucin-related outcomes. Unfortunately, in the 48 patients involved, mucin stores and gene expression were not significantly reduced in the treatment arm and there was additionally a dose-related increase in adverse events, including liver enzyme elevation and reduction in forced expiratory volume in $1 \mathrm{~s}$ [89].

Sadly, the effects of inhibiting the EGFR pathway appear to not be without consequences. It has been noted in lung cancer patients receiving TKIs there is a $1-5 \%$ incidence of interstitial lung disease depending on population group $[90,91]$. This TKI-dependent interstitial lung disease may be associated with the coadministration of radio- or chemotherapy [92], severe acute interstitial lung disease [92], older age, smoking and poor lung performance status [93]. These data suggest that improvements to EGFR pathway target identification, compound optimisation and patient selection remain warranted for chronic lung disease.

\section{Summary and future directions}

To date, antagonism of the EGFR pathway activity has only been shown to be clinically beneficial in treating lung cancer. This is most likely due to the significant complexity of the EGFR signalling pathway and demonstrates that further studies are needed to understand the pharmacokinetics, safety, and potential clinical efficacy of existing and in-development compounds for treating other chronic respiratory diseases with or without identified EGFR pathway mutations but with clear EGFR pathway hyperactivation (fig. 3). Despite this, the preclinical evidence that abnormal EGFR signalling drives respiratory pathology in a wide range of lung diseases in considerable. To overcome current challenges in targeting the EGFR pathway for therapeutic development, a comprehensive understanding of EGFR signalling coupled with careful compound selection and molecular targeting is needed. Future targets for therapy in chronic respiratory diseases where EGFR is hyperactivated are likely to include inhibition of ligand binding affinity, receptor homo- and/or heterodimerisation, pharmacological manipulation of ligand release and activation by metalloproteinase or other enzymes, restoration of physical segregation between EGFR ligands and receptors, enhanced avidity of intracellular and cell-surface antagonists for EGFR receptors, and a restriction of choice of downstream EGFR signalling pathways (fig. 2). Regardless of target, a key challenge for effectively antagonising EGFR signalling will be to minimise deleterious effects while maintaining normal respiratory functions.

\section{References}

1 Loddenkemper R, Gibson GJ, Sibille Y. European Lung White Book: The First Comprehensive Survey on Respiratory Health in Europe. Sheffield, European Respiratory Society, 2003.

2 Ask K, Eickelberg O, Gauldie J, et al. Have advanced research technologies made real impact on respiratory medicine? Respirology 2010; 15: 876-880.

3 Fingerlin TE, Murphy E, Zhang W, et al. Genome-wide association study identifies multiple susceptibility loci for pulmonary fibrosis. Nat Genet 2013; 45: 613-620.

4 Pleasance ED, Stephens PJ, O’Meara S, et al. A small-cell lung cancer genome with complex signatures of tobacco exposure. Nature 2010; 463: 184-190.

5 Soler Artigas M, Loth DW, Wain LV, et al. Genome-wide association and large-scale follow up identifies 16 new loci influencing lung function. Nat Genet 2011; 43: 1082-1090. 
Wilk JB, Shrine NR, Loehr LR, et al. Genome-wide association studies identify CHRNA5/3 and HTR4 in the development of airflow obstruction. Am J Respir Crit Care Med 2012; 186: 622-632.

Wills-Karp M, Ewart SL. Time to draw breath: asthma-susceptibility genes are identified. Nat Rev Genet 2004; 5: 376-387.

8 Lee AJ, Swanton C. Tumour heterogeneity and drug resistance: personalising cancer medicine through functional genomics. Biochem Pharmacol 2012; 83: 1013-1020.

9 Sharma SV, Bell DW, Settleman J, et al. Epidermal growth factor receptor mutations in lung cancer. Nat Rev Cancer 2007; 7: 169-181.

10 Burgel PR, Nadel JA. Epidermal growth factor receptor-mediated innate immune responses and their roles in airway diseases. Eur Respir J 2008; 32: 1068-1081.

11 Lai HY, Rogers DF. Mucus hypersecretion in asthma: intracellular signalling pathways as targets for pharmacotherapy. Curr Opin Allergy Clin Immunol 2010; 10: 67-76.

12 Yarden Y, Sliwkowski MX. Untangling the ErbB signalling network. Nat Rev Mol Cell Biol 2001; 2: $127-137$.

13 Zhang H, Berezov A, Wang Q, et al. ErbB receptors: from oncogenes to targeted cancer therapies. J Clin Invest 2007; 117: 2051-2058.

14 Graus-Porta D, Beerli RR, Daly JM, et al. ErbB-2, the preferred heterodimerization partner of all ErbB receptors, is a mediator of lateral signaling. EMBO J 1997; 16: 1647-1655.

15 Lemmon MA. Ligand-induced ErbB receptor dimerization. Exp Cell Res 2009; 315: 638-648.

16 Jorissen RN, Walker F, Pouliot N, et al. Epidermal growth factor receptor: mechanisms of activation and signalling. Exp Cell Res 2003; 284: 31-53.

17 Wilson KJ, Gilmore JL, Foley J, et al. Functional selectivity of EGF family peptide growth factors: implications for cancer. Pharmacol Ther 2009; 122: 1-8.

18 Higashiyama S, Iwabuki H, Morimoto C, et al. Membrane-anchored growth factors, the epidermal growth factor family: beyond receptor ligands. Cancer Sci 2008; 99: 214-220.

19 Sahin U, Weskamp G, Kelly K, et al. Distinct roles for ADAM10 and ADAM17 in ectodomain shedding of six EGFR ligands. J Cell Biol 2004; 164: 769-779.

20 Horiuchi K, Le Gall S, Schulte M, et al. Substrate selectivity of epidermal growth factor-receptor ligand sheddases and their regulation by phorbol esters and calcium influx. Mol Biol Cell 2007; 18: 176-188.

21 Prenzel N, Zwick E, Daub H, et al. EGF receptor transactivation by G-protein-coupled receptors requires metalloproteinase cleavage of proHB-EGF. Nature 1999; 402: 884-888.

$22 \mathrm{Yu}$ WH, Woessner JF Jr, McNeish JD, et al. CD44 anchors the assembly of matrilysin/MMP-7 with heparin-binding epidermal growth factor precursor and ErbB4 and regulates female reproductive organ remodeling. Genes Dev 2002; 16: 307-323.

23 Vermeer PD, Einwalter LA, Moninger TO, et al. Segregation of receptor and ligand regulates activation of epithelial growth factor receptor. Nature 2003; 422: 322-326.

24 Shaykhiev R, Zuo WL, Chao I, et al. EGF shifts human airway basal cell fate toward a smoking-associated airway epithelial phenotype. Proc Natl Acad Sci USA 2013; 110: 12102-12107.

25 Jensen KB, Watt FM. Single-cell expression profiling of human epidermal stem and transit-amplifying cells: Lrig1 is a regulator of stem cell quiescence. Proc Natl Acad Sci USA 2006; 103: 11958-11963.

26 Jensen KB, Collins CA, Nascimento E, et al. Lrig1 expression defines a distinct multipotent stem cell population in mammalian epidermis. Cell Stem Cell 2009; 4: 427-439.

27 Wong VW, Stange DE, Page ME, et al. Lrig1 controls intestinal stem-cell homeostasis by negative regulation of ErbB signalling. Nat Cell Biol 2012; 14: 401-408.

28 Lu L, Teixeira VH, Yuan Z, et al. LRIG1 regulates cadherin-dependent contact inhibition directing epithelial homeostasis and pre-invasive squamous cell carcinoma development. J Pathol 2013; 229: 608-620.

29 Zhang X, Pickin KA, Bose R, et al. Inhibition of the EGF receptor by binding of MIG6 to an activating kinase domain interface. Nature 2007; 450: 741-744.

30 Avraham R, Yarden Y. Feedback regulation of EGFR signalling: decision making by early and delayed loops. Nat Rev Mol Cell Biol 2011; 12: 104-117.

31 Goh LK, Huang F, Kim W, et al. Multiple mechanisms collectively regulate clathrin-mediated endocytosis of the epidermal growth factor receptor. J Cell Biol 2010; 189: 871-883.

32 Eden ER, Huang F, Sorkin A, et al. The role of EGF receptor ubiquitination in regulating its intracellular traffic. Traffic 2012; 13: 329-337.

33 Singh AB, Harris RC. Autocrine, paracrine and juxtacrine signaling by EGFR ligands. Cell Signal 2005; 17: $1183-1193$.

34 Roepstorff K, Grandal MV, Henriksen L, et al. Differential effects of EGFR ligands on endocytic sorting of the receptor. Traffic 2009; 10: 1115-1127.

35 Korfhagen TR, Swantz RJ, Wert SE, et al. Respiratory epithelial cell expression of human transforming growth factor-alpha induces lung fibrosis in transgenic mice. J Clin Invest 1994; 93: 1691-1699.

36 Miettinen PJ, Warburton D, Bu D, et al. Impaired lung branching morphogenesis in the absence of functional EGF receptor. Dev Biol 1997; 186: 224-236.

37 Le Cras TD, Hardie WD, Fagan K, et al. Disrupted pulmonary vascular development and pulmonary hypertension in transgenic mice overexpressing transforming growth factor-alpha. Am J Physiol Lung Cell Mol Physiol 2003; 285: L1046-L1054.

38 Madtes DK, Elston AL, Hackman RC, et al. Transforming growth factor-alpha deficiency reduces pulmonary fibrosis in transgenic mice. Am J Respir Cell Mol Biol 1999; 20: 924-934.

39 Plopper CG, St George JA, Read LC, et al. Acceleration of alveolar type II cell differentiation in fetal rhesus monkey lung by administration of EGF. Am J Physiol 1992; 262: L313-L321.

40 Finigan JH, Downey GP, Kern JA. Human epidermal growth factor receptor signaling in acute lung injury. Am J Respir Cell Mol Biol 2012; 47: 395-404.

41 Patel NV, Acarregui MJ, Snyder JM, et al. Neuregulin-1 and human epidermal growth factor receptors 2 and 3 play a role in human lung development in vitro. Am J Respir Cell Mol Biol 2000; 22: 432-440.

42 Liu W, Volpe MA, Zscheppang K, et al. ErbB4 regulates surfactant synthesis and proliferation in adult rat pulmonary epithelial cells. Exp Lung Res 2009; 35: 29-47. 
43 Zscheppang K, Dork T, Schmiedl A, et al. Neuregulin receptor ErbB4 functions as a transcriptional cofactor for the expression of surfactant protein B in the fetal lung. Am J Respir Cell Mol Biol 2011; 45: 761-767.

44 Zscheppang K, Liu W, Volpe MV, et al. ErbB4 regulates fetal surfactant phospholipid synthesis in primary fetal rat type II cells. Am J Physiol Lung Cell Mol Physiol 2007; 293: L429-L435.

45 Vermeer PD, Panko L, Karp P, et al. Differentiation of human airway epithelia is dependent on erbB2. Am J Physiol Lung Cell Mol Physiol 2006; 291: L175-L180.

46 LeSimple P, van Seuningen I, Buisine MP, et al. Trefoil factor family 3 peptide promotes human airway epithelial ciliated cell differentiation. Am J Respir Cell Mol Biol 2007; 36: 296-303.

47 Hardie WD, Bruno MD, Huelsman KM, et al. Postnatal lung function and morphology in transgenic mice expressing transforming growth factor-alpha. Am J Pathol 1997; 151: 1075-1083.

48 Andrianifahanana M, Wilkes MC, Gupta SK, et al. Profibrotic TGF $\beta$ responses require the cooperative action of PDGF and ErbB receptor tyrosine kinases. FASEB J 2013; 27: 4444-4454.

49 Ishii Y, Fujimoto S, Fukuda T. Gefitinib prevents bleomycin-induced lung fibrosis in mice. Am J Respir Crit Care Med 2006; 174: 550-556.

50 Takeyama K, Fahy JV, Nadel JA. Relationship of epidermal growth factor receptors to goblet cell production in human bronchi. Am J Respir Crit Care Med 2001; 163: 511-516.

51 O'Donnell RA, Richter A, Ward J, et al. Expression of ErbB receptors and mucins in the airways of long term current smokers. Thorax 2004; 59: 1032-1040.

52 Burgel PR, Montani D, Danel C, et al. A morphometric study of mucins and small airway plugging in cystic fibrosis. Thorax 2007; 62: 153-161.

53 Kohri K, Ueki IF, Shim JJ, et al. Pseudomonas aeruginosa induces MUC5AC production via epidermal growth factor receptor. Eur Respir J 2002; 20: 1263-1270.

54 Shao MX, Ueki IF, Nadel JA. Tumor necrosis factor alpha-converting enzyme mediates MUC5AC mucin expression in cultured human airway epithelial cells. Proc Natl Acad Sci USA 2003; 100: 11618-11623.

55 Blanchet S, Ramgolam K, Baulig A, et al. Fine particulate matter induces amphiregulin secretion by bronchial epithelial cells. Am J Respir Cell Mol Biol 2004; 30: 421-427.

56 Rumelhard M, Ramgolam K, Auger F, et al. Effects of PM2.5 components in the release of amphiregulin by human airway epithelial cells. Toxicol Lett 2007; 168: 155-164.

57 Rose MC, Piazza FM, Chen YA, et al. Model systems for investigating mucin gene expression in airway diseases. J Aerosol Med 2000; 13: 245-261.

58 Takeyama K, Dabbagh K, Lee HM, et al. Epidermal growth factor system regulates mucin production in airways. Proc Natl Acad Sci USA 1999; 96: 3081-3086.

59 Wills-Karp M. Interleukin-13 in asthma pathogenesis. Immunol Rev 2004; 202: 175-190.

60 Booth BW, Adler KB, Bonner JC, et al. Interleukin-13 induces proliferation of human airway epithelial cells in vitro via a mechanism mediated by transforming growth factor-alpha. Am J Respir Cell Mol Biol 2001; 25: 739-743.

61 Burgel PR, Lazarus SC, Tam DC, et al. Human eosinophils induce mucin production in airway epithelial cells via epidermal growth factor receptor activation. J Immunol 2001; 167: 5948-5954.

62 Val S, Belade E, George I, et al. Fine PM induce airway MUC5AC expression through the autocrine effect of amphiregulin. Arch Toxicol 2012; 86: 1851-1859.

63 Lynch TJ, Bell DW, Sordella R, et al. Activating mutations in the epidermal growth factor receptor underlying responsiveness of non-small-cell lung cancer to gefitinib. N Engl J Med 2004; 350: 2129-2139.

64 Paez JG, Janne PA, Lee JC, et al. EGFR mutations in lung cancer: correlation with clinical response to gefitinib therapy. Science 2004; 304: 1497-1500.

65 Meuwissen R, Berns A. Mouse models for human lung cancer. Genes Dev 2005; 19: 643-664.

66 Regales L, Gong Y, Shen R, et al. Dual targeting of EGFR can overcome a major drug resistance mutation in mouse models of EGFR mutant lung cancer. J Clin Invest 2009; 119: 3000-3010.

67 Ferby I, Reschke M, Kudlacek O, et al. Mig6 is a negative regulator of EGF receptor-mediated skin morphogenesis and tumor formation. Nat Med 2006; 12: 568-573.

68 Reschke M, Ferby I, Stepniak E, et al. Mitogen-inducible gene-6 is a negative regulator of epidermal growth factor receptor signaling in hepatocytes and human hepatocellular carcinoma. Hepatology 2010; 51: 1383-1390.

69 Hopkins S, Linderoth E, Hantschel O, et al. Mig6 is a sensor of EGF receptor inactivation that directly activates cAbl to induce apoptosis during epithelial homeostasis. Dev Cell 2012; 23: 547-559.

70 Jin N, Cho SN, Raso MG, et al. Mig-6 is required for appropriate lung development and to ensure normal adult lung homeostasis. Development 2009; 136: 3347-3356.

71 Mok TS, Wu YL, Thongprasert S, et al. Gefitinib or carboplatin-paclitaxel in pulmonary adenocarcinoma. $N$ Engl Med 2009; 361: 947-957.

72 Maemondo M, Inoue A, Kobayashi K, et al. Gefitinib or chemotherapy for non-small-cell lung cancer with mutated EGFR. N Engl J Med 2010; 362: 2380-2388.

73 Hanna N, Lilenbaum R, Ansari R, et al. Phase II trial of cetuximab in patients with previously treated non-small-cell lung cancer. J Clin Oncol 2006; 24: 5253-5258.

74 Kobayashi S, Boggon TJ, Dayaram T, et al. EGFR mutation and resistance of non-small-cell lung cancer to gefitinib. N Engl J Med 2005; 352: 786-792.

75 Jiang F, Qiu Q, Khanna A, et al. Aldehyde dehydrogenase 1 is a tumor stem cell-associated marker in lung cancer. Mol Cancer Res MCR 2009; 7: 330-338.

76 Huang CP, Tsai MF, Chang TH, et al. ALDH-positive lung cancer stem cells confer resistance to epidermal growth factor receptor tyrosine kinase inhibitors. Cancer Lett 2013; 328: 144-151.

77 Engelman JA, Zejnullahu K, Mitsudomi T, et al. MET amplification leads to gefitinib resistance in lung cancer by activating ERBB3 signaling. Science 2007; 316: 1039-1043.

78 Hirsh V. Afatinib (BIBW 2992) development in non-small-cell lung cancer. Future Oncol 2011; 7: 817-825.

79 Motzer RJ, Escudier B, Oudard S, et al. Efficacy of everolimus in advanced renal cell carcinoma: a double-blind, randomised, placebo-controlled phase III trial. Lancet 2008; 372: 449-456.

80 Madala SK, Schmidt S, Davidson C, et al. MEK-ERK pathway modulation ameliorates pulmonary fibrosis associated with epidermal growth factor receptor activation. Am J Respir Cell Mol Biol 2012; 46: 380-388. 
81 Le Cras TD, Korfhagen TR, Davidson C, et al. Inhibition of PI3K by PX-866 prevents transforming growth factoralpha-induced pulmonary fibrosis. Am J Pathol 2010; 176: 679-686.

82 Korfhagen TR, Le Cras TD, Davidson CR, et al. Rapamycin prevents transforming growth factor-alpha-induced pulmonary fibrosis. Am J Respir Cell Mol Biol 2009; 41: 562-572.

83 Simler NR, Howell DC, Marshall RP, et al. The rapamycin analogue SDZ RAD attenuates bleomycin-induced pulmonary fibrosis in rats. Eur Respir J 2002; 19: 1124-1127.

84 Malouf MA, Hopkins P, Snell G, et al. An investigator-driven study of everolimus in surgical lung biopsy confirmed idiopathic pulmonary fibrosis. Respirology 2011; 16: 776-783.

85 Cervantes F, Martinez-Trillos A. Myelofibrosis: an update on current pharmacotherapy and future directions. Expert Opin Pharmacother 2013; 14: 873-884.

86 Zhang J, Bi M, Zhong F, et al. Role of CIP4 in high glucose induced epithelial-mesenchymal transition of rat peritoneal mesothelial cells. Ren Fail 2013; 35: 989-995.

87 Yoshisue H, Hasegawa K. Effect of MMP/ADAM inhibitors on goblet cell hyperplasia in cultured human bronchial epithelial cells. Biosci Biotechnol Biochem 2004; 68: 2024-2031.

88 Li N, Li Q, Zhou XD, et al. Chronic mechanical stress induces mucin 5AC expression in human bronchial epithelial cells through ERK dependent pathways. Mol Biol Rep 2012; 39: 1019-1028.

89 Woodruff PG, Wolff M, Hohlfeld JM, et al. Safety and efficacy of an inhaled epidermal growth factor receptor inhibitor (BIBW 2948 BS) in chronic obstructive pulmonary disease. Am J Respir Crit Care Med 2010; 181: $438-445$.

90 Cohen MH, Williams GA, Sridhara R, et al. FDA drug approval summary: gefitinib (ZD1839) (Iressa) tablets. Oncologist 2003; 8: 303-306.

91 Takano T, Ohe Y, Kusumoto M, et al. Risk factors for interstitial lung disease and predictive factors for tumor response in patients with advanced non-small cell lung cancer treated with gefitinib. Lung Cancer 2004; 45: 93-104.

92 Teramoto S, Yamamoto H, Ouchi Y. Clinical efficacy and toxicity of gefitinib in patients with lung cancer. Lancet 2003; 361: 1992-1993.

93 Kudoh S, Kato H, Nishiwaki Y, et al. Interstitial lung disease in Japanese patients with lung cancer: a cohort and nested case-control study. Am J Respir Crit Care Med 2008; 177: 1348-1357. 